\title{
Power Margin Reduction in Linear passive UHF RFID tag arrays
}

\author{
Qi Zhang, Michael Crisp, Ian H. White and Richard V.Penty \\ Centre for Photonic Systems, Electrical Division, Department of Engineering, University of Cambridge, \\ 9 JJ Thomson Avenue, Cambridge, CB3 0FA, U.K.
}

\begin{abstract}
This paper studies the power margin reduction in linear passive UHF RFID tag arrays due to proximity effects. It is shown experimentally that a $40 \%$ reduction in tag power margin occurs when two tags are placed with a separation of less than $2 \mathrm{~cm}$. Major causes of tag sensitivity degradation due to proximity in arrays are analyzed by experiment and simulation, including tag detuning, shadowing and re-emission cancellation. It is shown that tag detuning has a significant effect when tags are separated by less than $7 \mathrm{~mm}$. At larger separations in excess of $1 \mathrm{~cm}$, the tag shadowing effect and interactions between the backscattered waves cause more significant degradation for large tag arrays.
\end{abstract}

Keywords-read range; close proximity; passive ultra high frequency (UHF); radio frequency identification (RFID); detuning; shadowing; re-emission cancellation

\section{INTRODUCTION}

In recent years, RFID technology has been used in a wide range of mainstream applications and passive UHF RFID tags are increasingly replacing current barcode systems due to their longer range, high data capacity, faster read speed and ability to be reprogrammed for repetitive use [1]. Passive UHF systems operate on the principle of backscatter communication with the tag antenna gathering energy from the electromagnetic wave transmitted by reader, and then using the energy to power a microchip. This then changes the load on the antenna to achieve backscatter modulation and communication back to the reader. RFID tags are usually attached to the surface of an object for identification purposes. If the object has significant dielectric properties or is conductive, the operation of the tag antenna is likely to be affected. For example, if an ordinary tag is placed next to a piece of metal, current induced in the metal generates fields which will interact with the antenna field often causing the tag's read range to drop significantly. As the tag antenna is made of metal, a similar situation can occur when tags are placed close to each other causing the tags to interact and the reader to fail to read one or both tags. Previously, it has been suggested that RFID tags need to be separated by around $10 \mathrm{~cm}$ between each other in order to be reliably detected successfully without significantly increasing the RF power [2]. This is clearly not practical for many applications such as item level tagging of small tightly packed objects.

Some experimental evaluations of the decrease in tag power margin due to interference between tags have been performed [3-4] and solutions have been proposed to improve tag operation in close proximity [5-7]. Yu Tanaka et al. [5] have found that tag detuning is the dominant effect when there are only two tags with a separation of less than $3 \mathrm{~cm}$. Detuning is caused by the power loss due to a mismatch between the tag antenna and IC, which originates from the impedance change of the tag antenna when one tag approaches another. Tanaka et al. proposed a solution to extend the tag read range by changing the RFID integrated circuit chip impedance to compensate for the impedance mismatch caused by the other tag antenna. Dobkin et al. [7] suggested that the effect of tag scattering plays an important role when multiple tags are placed in the close proximity. They have found that in a geometry in which the tags are more or less in the direction of the RF propagation, a tag shadowing behavior is observed and a more complicated situation arises when paths of scattered waves are not in the same direction as the incident waves. However, to the authors' knowledge, this is the first paper to analyze experimentally the ability to read tags in close proximity in tag arrays and to determine the dominant effects of tag power margin degradation. Three main factors namely, detuning, shadowing and re-emission cancellation, are analyzed, the first being analyzed using 2 tags, and the others being studied for tag arrays. We find that degradation due to re-emission from tags acting as coupled reflector arrays has a more significant effect when tags are placed with separations of more than $1 \mathrm{~cm}$.

\section{TWO TAGS IN CLOSE PROXIMITY}

To investigate the coupling effect when passive UHF RFID tags are placed in close proximity, an initial experiment is carried out with two tags placed in front of a single pair of antennas. The degradation in power margin caused by moving one tag with respect to the other is studied. The experimental arrangement is shown in Fig. 1. A bi-static antenna configuration with a separation of $1 \mathrm{~m}$ between the transmitting and receiving antennas ( $\mathrm{Tx}$ and $\mathrm{Rx}$ ) is used so that sufficient isolation is afforded for the experiments. The antennas are circularly polarised antennas with $6 \mathrm{dBi}$ gain and 70 degree beam width operating in the ETSI (European Telecommunications Standards Institute) frequency band of $865-867 \mathrm{MHz}$. The experiment is repeated twice with two different kinds of passive UHF RFID tags which have different antenna and IC designs but similar architecture. The first pair is UMP DogBone ${ }^{\mathrm{TM}}$ inlays using Impinj Monza 4 ICs and the second pair is Avery Dennison AD-232iL tags which use NXP uCode G2iL ICs. Both tags are effectively a short wide loaded dipole with a T-match as shown in Fig. 2. 


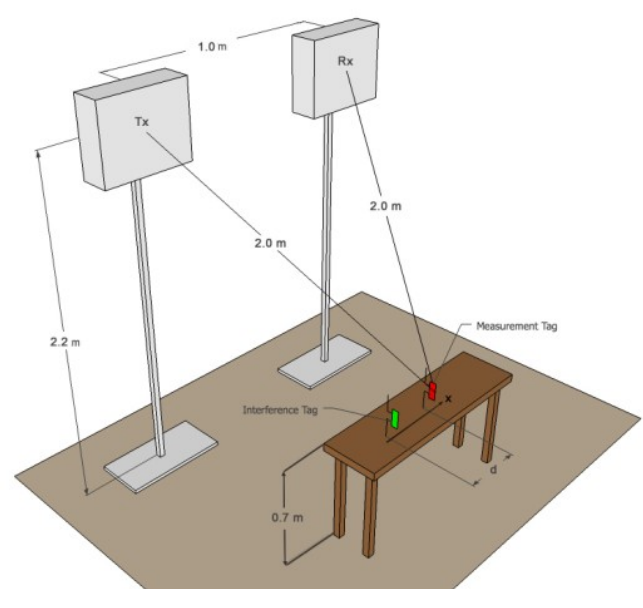

Fig. 1 Experiment arrangement

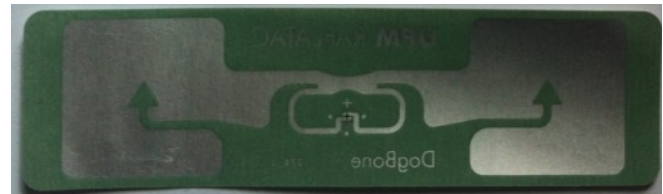

(a) Impinj Monza 4 tag

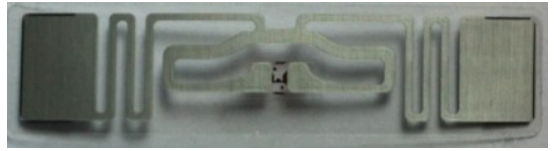

(b) $\mathrm{AD}-232 \mathrm{iL}$ tag

Fig. 2 Impinj Monza 4 tag and AD-232iL tag

The red tag shown in Fig. 1 is the measurement tag and is kept in the same position throughout the experiment. The green tag is moved to vary its interference with the measurement tag, and its position is varied to allow separations from $50 \mathrm{~cm}$ to $0 \mathrm{~cm}$ (i.e. touching the red tag). The transmitting and receiving antennas are located at a distance of $2 \mathrm{~m}$ from the measurement tag.

The reader is programmed to vary the transmission power from $36 \mathrm{dBm}$ to $22 \mathrm{dBm}$ EIRP at a fixed transmission frequency of $865.1 \mathrm{MHz}$. In order to reduce the impact of multi-path effects from the environment, the measurement tag is measured separately in isolation without the presence of the other tag before measuring with both of the tags present. The minimum transmission power needed for the reader to detect the tag successfully is recorded. The read power margin for each tag is defined as the ratio in $\mathrm{dB}$ of the maximum allowed transmission power and the minimum power needed for successful detection. The read power margin measured when both tags are present is subtracted from the power margin measured with only the measurement tag present. In this way, we exclude most of multipath effects, even though the interference tag could cause a double resonance effect, and only look at the change in read power margin at our target tag due to the interference from the other tag placed in close proximity. The same experimental procedure is repeated for the two different pairs of RFID tags. Fig. 3 presents a plot of the normalised power margin compared with that of isolated tags against the separation distance between tags. Fig. 4 shows the normalised power margin compared with that of isolated tags at close separation distances less than $35 \mathrm{~mm}$.

The reverse link margin also is measured by adding an attenuator after the receiver antenna and increasing the attenuation until the tag fails to read. Here the transmitting antenna is set at its maximum power allowable, $36 \mathrm{dBm}$ EIRP [8]. The measured received signal strength (RSSI) against attenuation is shown in Fig. 5. It is found that the reverse link margin for Impinj Monza 4 tag is $28 \mathrm{~dB}$ and for AD-232iL tag is $26 \mathrm{~dB}$, both of which are much larger than the maximum change in transmission power of $14 \mathrm{~dB}$ in the experiment ensuring that the system is forward link limited.

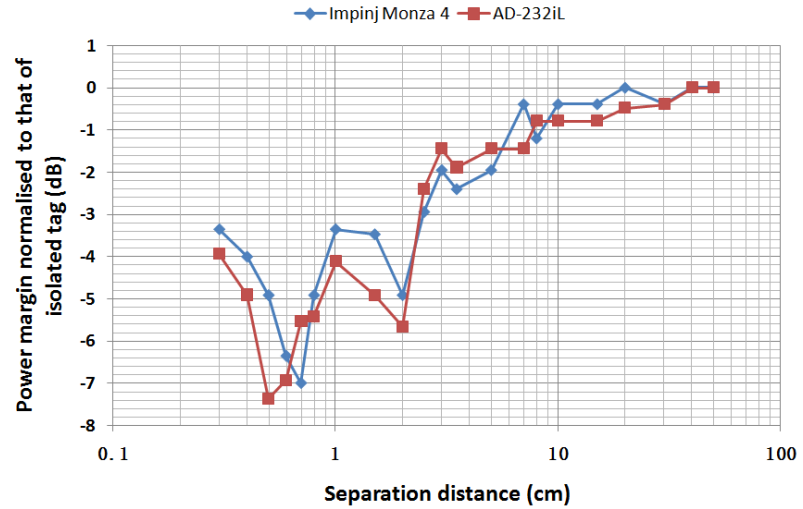

Fig. 3 Normalised power margin compared with that of isolated tags plotted as a function of separation reduced.

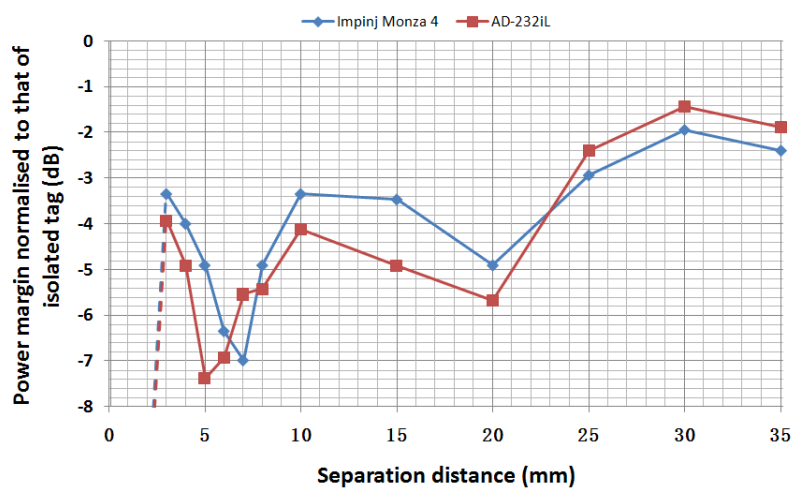

Fig. 4 Normalised power margin compared with that of isolated tags at close separation distances

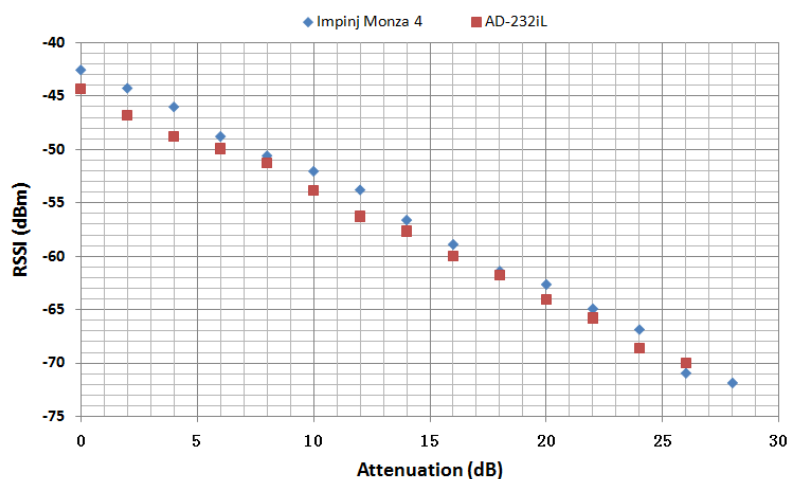

Fig. 5 Reverse link measurement

It is observed that in Fig. 3 both Impinj Monza 4 tags and $\mathrm{AD}-232 \mathrm{iL}$ tags have a similar performance when two tags are placed in close proximity. Several trends can be 
observed in terms of read power margin versus tag separation. Firstly, the read power margin does not change significantly when the separation is greater than $10 \mathrm{~cm}$. Secondly as the interference tag approaches the measurement tag with a separation less than $10 \mathrm{~cm}$, the power margin reduces as the tag interference becomes stronger. When two tags are placed very close to each other, at $5-7 \mathrm{~mm}$ separation for example, approximately $7 \mathrm{~dB}$ more transmission power is needed to detect the tag than when the two tags are separated at distances greater than $10 \mathrm{~cm}$. It is thirdly observed that at a separation less than $5 \mathrm{~mm}$, the power margin for the measurement tag fluctuates. This is studied in more detail later in the paper. Finally, it should be noted that when the two tags are almost touching with a separation of less than $3 \mathrm{~mm}$, the measurement tag cannot be detected by the reader even when using the maximum transmission power. In the following sections, the key causes of these trends are considered.

\section{TAG DETUNING}

Impedance matching between the antenna and the tag IC is particularly critical to achieve sufficient power into the tag to activate the IC. Often a conjugate match is chosen to maximise power transfer. This is further complicated by the non-linear tag load characteristic and wide bandwidth required to enable global operation. Passive UHF RFID tag antennas are often short dipoles with a matching loop based on a T-matching technique to couple power into the integrated circuit [9]. At its resonant frequency, the induced voltage across the tuned tag is significantly enhanced compared with that for frequencies outside the resonant range. This resonance causes an increased tag read power margin at the design frequency.

Tag detuning can be caused by the changes in the antenna impedance when the tag is placed on an object or when other objects are present in the vicinity of the tag due to their mutual inductances [10]. The voltage induced in the antenna of the tag by the magnetic field from the neighbouring tags can cause a shift in the resonant frequency (detuning). Once the tag antenna is detuned, the energy from the reader interrogation field cannot be efficiently coupled into the RFID tag and hence a decrease in its maximum read distance results [10].

To examine the significance of tag detuning to the problem of detecting tags in close proximity, the change in the antenna impedance is directly measured as one tag approaches another tag. Xianming Qing et al. [11] have illustrated a method of extracting the balanced RFID antenna impedance directly from measured single ended Sparameters. This method is used here to measure the change in impedance on one tag as another tag is placed in close proximity. The schematic configuration of the experiment is given in Fig. 6. The measurement is carried out by using a two port VNA and a test fixture. The test fixture is built by soldering the outer conductor of two $10 \mathrm{~cm}$ semi-ridged coaxial cables together. One end of the fixture with SMA connectors is used to connect to the VNA. The other end of the fixture is open with a small extension from the inner coaxial conductor to connect the test tag antenna [11]. The test antenna is obtained by carefully removing the microchip from the tag. The impedance of the tag antenna is calculated by measuring the S-parameter from the VNA. Conventional calibration is carried out initially on the electrical plane before the SMA connectors on the test fixture, and then a port extension is added with the test fixture shorted until an ideal short is observed on a smith chart display on the VNA. The short is then replaced with the antenna under test. Another identical tag (complete with IC) is used as an interference tag. Offline processing of the recorded complex S11 and S21 allows extraction of the antenna impedance. The measurement is taken at different separation distances as the interference tag is moved closer to the test antenna in the $\mathrm{x}$ direction as indicated in Fig. 6. The measurement tag is kept at the same position throughout the experiment. The $\mathrm{AD}-232 \mathrm{iL}$ tags are used for this measurement. The interference tag is fixed to a foam stand and micro manipulator used to accurately change its position and distance from the measurement tag.

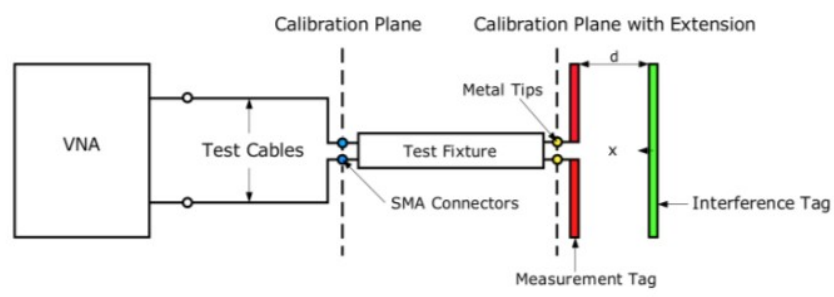

Fig. 6 Schematic configurations for measuring antenna impedance

Fig. 7 shows the calculated impedance for the test antenna based on measured S-parameters as the separation distance between the tags is varied. The dotted line indicates the impedance of the test antenna when it is measured in isolation. Fig. 8 illustrates the calculated change in power delivered to test tag's IC and power back scattered based on the methodology found in [5].

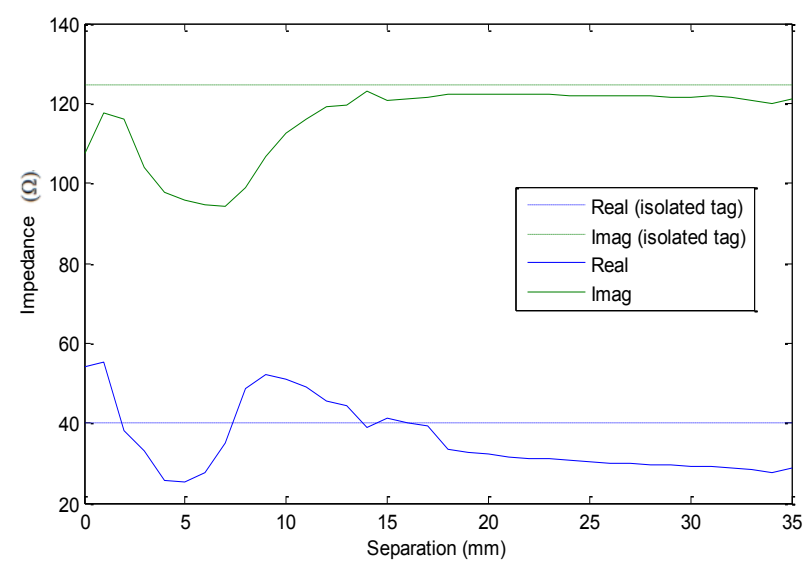

Fig. 7 Impedance measured for testing antenna against separation distances 


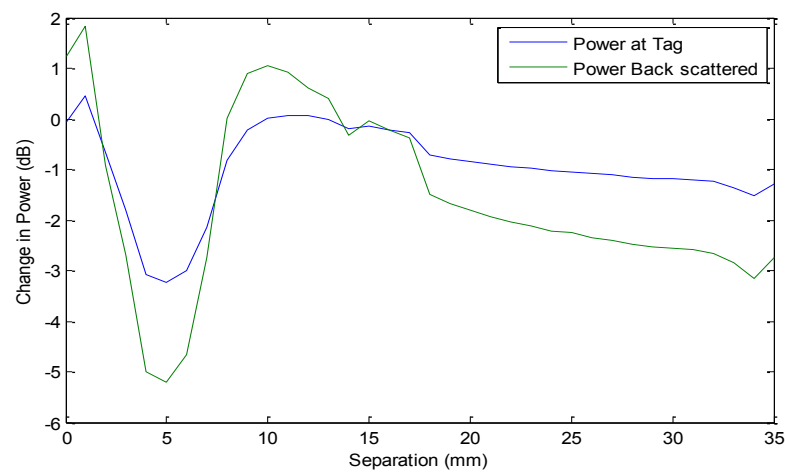

Fig. 8 Change in power received at tag/back scattered against separation distances

It can be seen that, at a separation distance around $5 \mathrm{~mm}$, the experimental measurement in Fig. 8 shows a peak power margin reduction which correlates well with measurement results for $\mathrm{AD}-232 \mathrm{iL}$ tag in Fig. 4 in Section II. This indicates that at very close tag separation, the impedance mismatch is significant in causing the reduction in tag read power margin. However, it is also noticed that the peak reduction in Fig. 8 is around 5dB, whereas in Fig. 4, a larger reduction of around $7 \mathrm{~dB}$ is observed. This indicates that other effects must also be causing degradation, which must become more significant to cause even greater margin reduction at very small separation. In addition, with a separation distance greater than $1 \mathrm{~cm}$, the change in power received at the tag and the back scattered power varies by only 1 to $2 \mathrm{~dB}$. This is much smaller than the change in tag power margin observed in the experimental results in Section II where large changes of more than $3 \mathrm{~dB}$ are observed when the separation distance are much larger (1-3 $\mathrm{cm})$. This implies the impedance mismatch becomes less significant when tags have a separation greater than $7 \mathrm{~mm}$ and the reduction of read power margin of passive UHF tags observed in Section II is not only due to the impedance mismatch of the tag caused by detuning at separations greater than $7 \mathrm{~mm}$.

\section{PROXIMITY EFFECTS FOR TAG ARRAYS}

\section{A. Tag shadowing}

It is observed from the experiment results in Section II that even when tags are separated by relatively large distances from each other $(2 \mathrm{~cm})$, the interaction between neighbouring tags is still substantial. One other possible reason for this is the shadowing effect: when tags are linearly aligned, the tags nearest to the interrogating antenna capture energy from a reader resulting in less available energy for tags further away [7]. The assessment of this is difficult using only two tags and so here an array experiment is carried out.

This experiment tests an array of 57 RFID tags which are spaced at $1 \mathrm{~cm}$ intervals. The same antennas in Section II are used. The tags are Impinj Monza 4 tags. Fig. 9 shows the plan view of the experiment arrangement. The red and green antennas indicate the transmitting and receiving antennas respectively. As in previous experiments, the transmission power is reduced from $36 \mathrm{dBm}$ EIRP to $22 \mathrm{dBm}$ EIRP. The minimum transmission power needed to detect each tag within the array is recorded and plotted against tag position in the array shown as Fig. 10. The tag array is placed at a distance of $2.5 \mathrm{~m}$ from the antennas. The measurements are taken with two different tag array orientations: firstly the tag array is positioned such that the long axis of the array is orthogonal to the interrogator wave fronts with tag ID 57 closest to the antennas (shown as 90 degree orientation in Fig. 9) and then the array is rotated 90 degree so that its long axis is parallel to the interrogator wave fronts (shown as 0 degree orientation in Fig. 9).

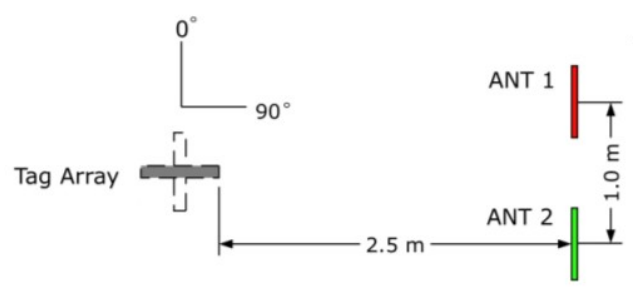

Fig. 9 Experiment arrangement for tag array orientation test

The blue points in Fig. 10 show the results when tag array is placed at an orientation with the long array axis orthogonal to the interrogator wave fronts. In the results, a minimum detection power of $36 \mathrm{dBm}$ is used to indicate that the tag cannot be detected by the reader even at maximum output power. This is because the maximum transmission power from the reader is limited by regulations, usually set to be $36 \mathrm{dBm}$ EIRP [8]. It is observed that in general, the tags close to the transmitting antenna perform better than the tags further away. This is indicated in Fig. 10 that the first half of the array (tag ID from 57 to 30) in general requires less transmission power for successful detection than the second half of the array (tag ID 29 to 1). However, if the tag shadowing is the only significant effect in the proximity problem, it would be expected that a simple increasing trend from tag ID 57 (the tag closest to the reader antenna) to tag ID 1 (the tag at the furthest distance from the reader antenna) in the minimum transmission power needed for sucessful detection would be seen. However, it is seen that the points do not follow a simple increasing trend, especially in the second half of the array (tag ID 29 to 1) which features an unexpected decreasing trend. The tag at the furthest end of the array does not require the greatest transmission power from the reader.

Comparing the results for the other orientation of the array where the long axis is parallel to the interrogator wave fronts (shown as 0 degree orientation in Fig. 9), if tag shadowing were the only significant effect, one would expect that all tags in the array would have the same read power margin in this configuration and that the margin would be increased compared to the orthogonal orientation since there would be effectively no shadowing. Clearly this is not the case, and in fact the reverse is seen, especially at the first half of the array (tag ID 57 to 30) where test results from 90 degree orientation clearly outperforms that of 0 degree orientation. Therefore, tag shadowing is not the only dominant effect in close proximity in an tag array. 


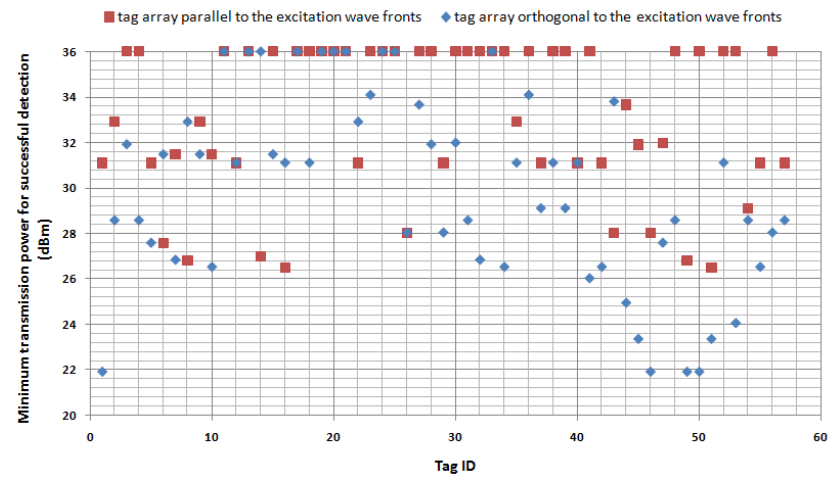

Fig. 10 Experiment result for tag array directionality

\section{B. Tag re-emission cancellation}

Tag re-emission cancellation is another important factor to consider in detecting tags in an array. The problem is caused by the re-emitted waves from the tags and the excitation wave from the reader combining in a manner which leads to an interference pattern. In certain locations the interference will be destructive and prevent tag reading. It is difficult to test this directly through experiment as other multipath effects play a significant role in real environments which have unwanted reflections and distortions. However the measurement of directionality as shown in Fig. 10 is a strong indicator that tags are acting in a similar manner to the passive elements of a Yagi-Uda antenna.

FEKO Lite, an electromagnetic simulation software based on the method of moments (MoM) technique is used to simulate a tag array to study the effect in more detail. As shown in Fig. 11, a simple wire model of a passive UHF RFID tag is created approximating the tag to a folded dipole antenna with a T-match. The microchip of the tag is modelled as a complex load impedance, which is set to be the conjugate match of the tag antenna. The tag is optimised to work at operating frequency of $865.1 \mathrm{MHz}$.

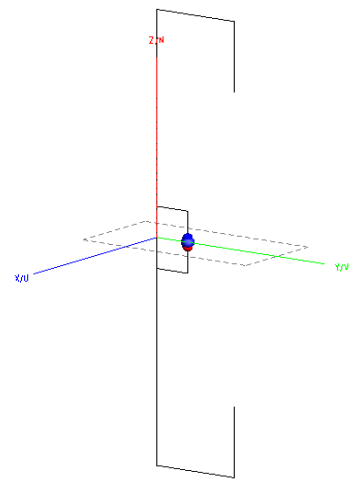

Fig. 11 Model of a single tag

An array of 57 tags is created in the model with a separation distance of $1 \mathrm{~cm}$ between neighbouring tags as shown in Fig. 12. The excitation wave is circularly polarised at a frequency of $865.1 \mathrm{MHz}$ and has a field strength of $1 \mathrm{~V} / \mathrm{m}$. Tests are carried out for two directions of the excitation wave: from one end of the array and from the side of the array respectively as shown in Fig. 12 below. For each case, the power received by the load (which represents the IC) for each tag is recorded and plotted against its position in the array as shown in Fig. 13.

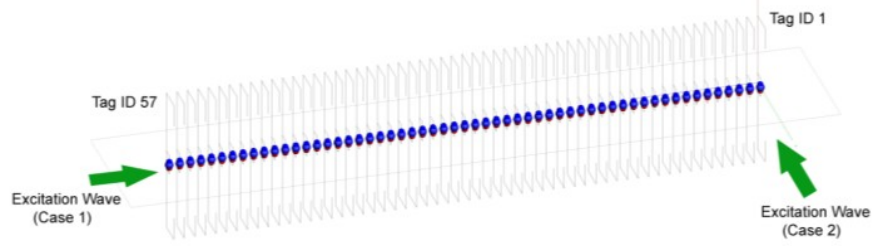

Fig. 12 Model of the tag array

The simulation result agrees in general terms with the experiment showing the directionality of the tag array in Fig. 10 in Section IV. It is noticed that for the red curve in Fig. 13 when the tag array is placed at the orientation parallel to the excitation wave fronts, almost every tag in the array receives less energy compared with the blue curve when the tag array has its long axis orthogonal to the excitation wave fronts in the first half of the tag array (tag ID 57 to 30). In many cases, the decrease in power into the IC is significant.

It is seen in Fig. 13 that, as indicated by the blue line, the power captured by the IC does not simply follow a decreasing trend (as it would if shadowing was dominant). The tag closest to the radiation source (tag ID 57) indeed has the highest captured energy compared with other tags in the array and the curve initially follows a decreasing tend until about half way through the array. The second half of the array however, shows a cyclical behaviour and a slowly increasing trend in the end of the array There is a $16 \mathrm{~dB}$ increase in the power received by the IC in the furthest tag (tag ID 1) compared with the tag which captures the least amount of energy in the array (tag ID 24). Indeed this simulation result agrees qualitatively with the features in the experimental result in Fig. 10. Close agreement is not expected due to multipath effects not taken into account in the simple model.

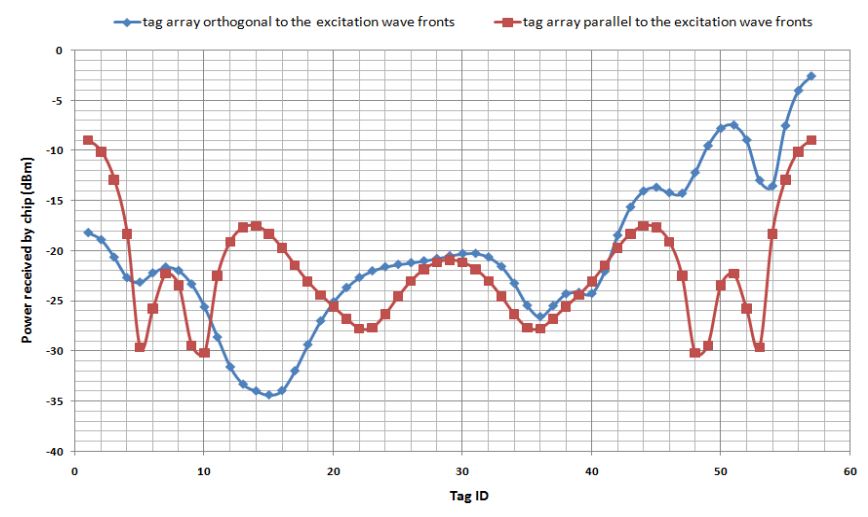

Fig. 13 Power into chip (in $\mathrm{dBm}$ ) against tag ID

It is observed that the general shape of the blue curve in Fig. 13 is very similar to the pattern of field penetration in a distributed Bragg reflector used in optical devices [12]. The structure of the distributed Bragg reflector consists of an alternating sequence of two materials of different optical impedances which can be treated as being similar to a tag array with air gaps between neighbouring tags. 


\section{CONCLUSION}

In this paper, we have experimentally demonstrated a $40 \%$ read power margin reduction when two tags are placed at close separations of less than $2 \mathrm{~cm}$. We have also analysed the dominant effects causing read power margin reduction in conventional passive UHF RFID tags placed in large linear arrays taking into account tag detuning, tag shadowing and re-emission cancellation. It is found that different effects operate over different length scales of separation. Tag detuning has a significant effect when tags are placed very close to one another (at $7 \mathrm{~mm}$ separation) and has less influence when tags are placed with separation distance greater than $1 \mathrm{~cm}$. For long arrays of tags, tag shadowing is found to be not the only significant effect when tags are placed in an array in close proximity. Interaction between the backscattered waves causes an interference pattern which also has a significant effect in tag array with separations as low as $1 \mathrm{~cm}$ which leads to a significant reduction in the read range of some RFID tags in the array. The effect causes a strong directionality in the tag array. The resulting interference pattern is similar to those observed in other systems of multiple reflectors such as Bragg gratings.

\section{ACKNOWLEDGEMENT}

This work has been supported by UK Engineering and Physical Science Research Council via the COPOSII project.

\section{REFERENCES}

[1] Klaus Finkenzeller, RFID Handbook: Fundamentals and Applications in Contactless Smart Cards and Identification, $2^{\text {nd }}$ edition, New York, NY USA: Wiley, 2003, pp. 29-180, pp. 229-328.
[2] United States Department of Defense Suppliers' Passive RFID Information Guide, Version 15.0, pp. 20, [Online]. Avilable: http://www.acq.osd.mil/log/sci/ait/DoD_Suppliers_Passive_RFID_Inf o_Guide_v15update.pdf.

[3] Steven M.Weigand and Daniel M.Dobkin, "Multiple RFID Tag Plane Array Effects", IEEE Antennas and Propagation Society Int. Symp., 2006, pp. 1027-1030.

[4] D. B. Dobkin, and S. M. Weigrand, "UHF RFID and Tag Antenna Scattering, Part I: Experimental Results," Microwave Journal, May 5, 2006, vol. 47, no. 5, pp. 170.

[5] Yu Tanaka, Yohtaro Umeda, Osamu Takyu, Machiko Nakayama and Kazunari Kodama, "Change of read range for UHF passive RFID tags in close proximity", IEEE Int. Conf. RFID, 2009, pp. 338-345..

[6] J. Lorenzo, D. Girbau, A. Lazaro and R. Vilarino, "Read range reduction in UHF RFID due to antenna detuning and gain penalty", Microwave and Optical Tech. Letters, 2011, vol. 53, no. 1, pp. 144148.

[7] Daniel Dobkin, "Nearby tags", The RF in RFID Passive UHF RFID in Practice, Oxford, UK: Elsevier, 2008, pp. 345-346.

[8] Daniel D.Deavours, UHF EPC Tag Performance Evlaution, [Online]. Avilable: http://www.rfidjournal.net/ Alliance_Lab_Report_intro.pdf.

[9] Naaser A.Mohammed, Kenneth R.Demarest and Daniel D. Deavours, "Analysis and synthesis of UHF RFID antenna using the embedded T-match", IEEE Int. Conf. RFID, 2010, pp. 230-236.

[10] C. Floerkemeier and M. Lampe, "Issues with RFID usage in ubiquitous computing applications," $2^{\text {nd }}$ Int. Conf. PERVASIVE, 2004, pp. $188-193$.

[11] Xianming Qing, Chean Khan Goh and Zhi Ning Chen, "Impedance characterization of RFID tag antennas and application in tag codesign", IEEE Transactions on Microwave Theory and Techniques, 2009 , vol. 57 , no.5, pp. 1268-1274.

[12] Alexey Kavokin, Jeremy J.Baumerg, Guillaume Malpurch and Fabrice P.Laussy, Microcavities, New York, Clarendon Press, 2007, pp. 9-11. 